\title{
Dual formulations of non-abelian spin models: local representation and low-temperature asymptotics
}

\author{
O. Borisenko ${ }^{1}$, V. Kushnir ${ }^{2}$ \\ N.N.Bogolyubov Institute for Theoretical Physics, National Academy \\ of Sciences of Ukraine, 03143 Kiev, Ukraine
}

\begin{abstract}
Non-abelian lattice spin models with symmetry group $S U(N)$ or $U(N)$ can be formulated in terms of link variables which are subject to the Bianchi constraints. Using this representation we derive exact and local dual formulation for the partition function of such models on a cubic lattice in arbitrary dimension $D$. Locality means that the dual action is given by a sum over some subset of hypercubes of the dual lattice and the interaction between dual variables ranges over one given hypercube. Dual variables are in general discrete-valued and live on $(D-2)$-cell of the dual lattice, in close analogy with the $X Y$ model. We use our construction to study in details the dual of $S U(2)$ principal chiral model in two dimensions. We give dual expressions also for two-point correlation function in arbitrary representation and for the free energy of defects. Leading terms of the asymptotic expansion of the dual Boltzmann factor are computed and it is proven that at low temperatures it converges to a certain Gaussian distribution uniformly in all fluctuations of dual variables. This result enables us to define the semiclassical limit of the dual formulation and to determine an analog of the vortex-spinwave representation for the partition function. Such representation is used to extract leading perturbative contribution to the correlation function which shows power-like decay at weak coupling. We also present some analytical evidences that the low-temperature limit of the dual formulation is completely described by $I S O(2)$-like approximation of $S U(2)$ matrix elements.
\end{abstract}

\section{Introduction}

Two-dimensional $(2 D)$ lattice spin models, besides being interesting on their own right as models of ferromagnets can be viewed as mathematically well defined scheme of the nonperturbative regularization of quantum continuum field theories like $S U(N)$ principal chiral model or $O(N)$ nonlinear sigma model. Being a discrete theory lattice models can be simulated on computers by Monte-Carlo method, and this is nowadays the most important tool of obtaining physical results near the continuum limit. Among major analytical methods one could mention the strong coupling expansion and the perturbation theory (PT). The PT is essentially the only analytical tool which provides systematic expansion of different physical quantities in the region of weak coupling, i.e. in the region relevant for the construction

\footnotetext{
${ }^{1}$ email: oleg@bitp.kiev.ua

${ }^{2}$ email: vkushnir@i.kiev.ua
} 
of the continuum limit. However, at the best PT is only applicable for studying short-distance quantities and the most important and interesting phenomena, like the mass gap generation cannot be described in its frameworks. When attempting to go beyond PT in the region of weak bare coupling, one runs into various mathematical problems, the most important being an absence of any analytical control over exponentially small contributions and, hence the absence of any reliable method to study long-distance physics where nonperturbative effects dominate even if they are exponentially suppressed. These facts impelled people to look for different, though equivalent representations which would allow to study the long-distance physics. One of such popular and promising representations is known as a dual formulation and is based on a certain non-classical change of variables. This formulation deals with dual lattice and appears to be very fruitfull for abelian models [1]. Namely, within such formulation 1) have been obtained various important analytical results on the long-distance dynamics of abelian spin fields and 2) the topological structure of the vacuum is transparent and can be more easily studied.

In the context relevant to this paper we would like to mention the dual of the abelian $X Y$ model [1] which has been used to prove the existence of a soft phase at low temperatures with power-like decay of the correlation function in twodimensional $X Y$ model [2]. In this case the dual of the $X Y$ model is a local theory for certain discrete variables. The conventional dual transformations [3] for nonabelian models are perfectly well defined in mathematical terms and also lead to a local dual theory for integers which label irreducible representations of the symmetry group. Nevertheless, these transformations are not complete if one compares to the abelian case. First of all, the resulting dual variables are not independent but are subject to some constraints known as triangular conditions, and as such they cannot really be associated with elements of a dual lattice (of course, introduction of the dual lattice is not the crucial point but rather matter of convenience; e.g., in the abelian case one can work with dual variables on the original lattice). It is to say, however that it is not really clear what are true independent degrees of freedom of the duals of non-abelian models. Secondly, such formulation is so mathematically involved that today it is even far from obvious if it can be useful for any kind of the study of the model, except for the strong coupling expansion. In particular, the positivity of the Boltzmann weight remains unclear (see discussion in [3). Even the precise definition of what can be termed the dual Boltzmann weight is not really obvious, so the possibility of numerical simulations is at present rather uncertain. Most importantly, however is that it is not clear how one could proceed in an analytical study of the model at low-temperatures ${ }^{3}$.

On the other hand, there exists representation of $S U(N)$ and $U(N)$ spin models in terms of link variables [4, and this representation can be formulated directly on the dual lattice. It is a first goal of the present paper to use the link representation for the derivation of an exact dual formulation of non-abelian spin models. Here we give two such formulations which appear to be quite different from the conventional formulation mentioned above. In our opinion the most essential advantages of our

\footnotetext{
${ }^{3}$ This is, of course personal opinion of the authors. After some attempts to understand lowtemperature properties of the dual Gibbs measure we cannot say whether it possesses any kind of well defined asymptotic expansion at large $\beta$.
} 
formulations are that 1) one of them can be definitely used for the Monte-Carlo simulations in $2 D$ and 2) they are much more suitable for an analytical investigation of the model in the low-temperature region. The second fact follows from the properties of the link formulation and we refer for the details of why it is so to our papers [5, 6]. In the last of those papers we have already presented a dual of $2 D$ $S U(2)$ spin model and proposed approximate representation for the dual partition function at low temperatures. Another important feature of one of our formulations is that it carries a close analogy with the abelian $U(1)$ model. For instance, for $2 D S U(2)$ model there are 3 independent degrees of freedom per (dual) site. At low temperatures it possesses an analog of the vortex-spin-wave representation, etc. The deficiency of our approach is that it can be straightforwardly applied only to models in which spins are elements of the Lie group. We do not know any obvious generalization of link formulation to, say, $O(N)$ nonlinear sigma models.

Low-temperature properties of $2 D$ non-abelian models are crucial for construction of their continuum limit. It is commonly recognized that models possess no phase transition, correlation function has exponential decay at any coupling and models are asymptotically free. Despite being more than twenty five years old this expectation has not been proven rigorously. On the contrary, certain percolation theory arguments suggest that all non-abelian models have soft low-temperature phase with power-like decay of the correlation function [7. It is thus another important motivation of the present investigation, namely to get deeper insight into the nature of the mass gap in non-abelian spin models. For example, in many papers devoted to $2 D$ non-abelian models it is written that "there is a nonperturbative mass gap generation at arbitrarily small couplings". It is not really clear, however what is precise meaning of this "nonperturbative generation". It cannot be a simple consequence of the link decorrelation which happens in $1 D$ models. Then, one could ask if this "nonperturbative generation" follows from the existence of some non-trivial background of defects like vortices of the $X Y$ model or is due to the strong but smooth disorder of non-abelian spins, e.g. like center vortices [8. As is well known, the dual formulation of abelian models have been extremely useful in clarifying all these important physical problems [2, 9, 10]. We think that the dual formulation given here is a good starting point for obtaining reliable analytical results in the low-temperature limit of non-abelian models. This is our next goal to develop a technique within dual formulation of non-abelian models which would allow to investigate them in the limit of the weak coupling. In this context we study here the following two approaches on the simplest example of $S U(2)$ spin model

- We derive an asymptotic expansion of the dual Boltzmann weight when $\beta \rightarrow \infty$ uniformly valid in fluctuations of the dual variables. The same procedure is also done for the two-point correlation function. As will be seen, at low temperatures the Boltzmann weight converges to a certain Gaussian ensemble the fact which ensures a possibility to define an analog of the vortex-spin-wave representation for the partition and correlation functions in the semiclassical limit. This step provides a major simplification of the model as $\beta \rightarrow \infty$ but even in this case it cannot be yet solved exactly. The formulation obtained permits very simple calculation of the leading perturbative contribution to the 
correlation function. This contribution results in the power-like decay of the correlation function.

- The second approach consists in replacing $S U(2)$ matrix elements by $I S O(2)$ ones in the vicinity of the identity element of $S U(2)$. We shall give a proof that such replacement is valid at low temperatures, compute the asymptotics of the dual Boltzmann weight and present simple numerical evidence that practically for all configurations this way of calculations produces very reasonable approximation for the original Boltzmann weight.

This paper is organized as follows. In the section 2 we review briefly the conventional dual transformations. After introduction of the link formulation we develop original approach to the dual transformations and present two forms of the duals of the principal chiral model. In the section 3 we investigate the low-temperature properties of the dual Boltzmann weights of $S U(2)$ model in two dimensions along the lines described above. Some technical details of this investigation are given in the appendix. In the section 4 we summarize our results and outline some perspectives for future investigations. Also, we calculate here the leading perturbative contribution to the correlation function.

\section{Principal chiral models and their duals}

We work on a $D$-dimensional hypercubic lattice $\Lambda \in Z^{d}$ with lattice spacing $a=1$ and a linear extension $L$. We impose either free or periodic boundary conditions (BC). Let $G=U(N), S U(N) ; U(x) \in G$ and $D U(x)$ denote the Haar measure on $G$. $\chi_{r}(U)$ and $d(r)$ will denote the character and dimension of irreducible representation $\{r\}$ of $G$, correspondingly. We treat models with local interactions, i.e. interactions between nearest neighbours. Let $H[U]$ be a real invariant function on $G$ such that

$$
|H[U]| \leq H(I)
$$

for all $U$ and coefficients of the character expansion of $\exp (\beta H)$

$$
C[r]=\int D U \exp (\beta H[U]) \chi_{r}(U)
$$

exist. The partition function (PF) of the principal chiral model with the symmetry group $G \times G$ is defined as

$$
Z_{\Lambda}(\beta)=\int \prod_{x \in \Lambda} D U(x) \exp \left[\beta \sum_{x \in \Lambda} \sum_{n=1}^{D} H\left[U(x) U^{\dagger}\left(x+e_{n}\right)\right]\right] .
$$

\subsection{Standard dual transformations}

Conventional dual transformations can be defined as a sequence of transformations consisting of the following steps: 
1. Fourier expansion of the Boltzmann weight $\exp (H)$. This is an essentially character expansion on the group.

2. Exact integration over original degrees of freedom. As a result one obtains a set of constraints on the summation variables (which label representations of the group $G$ and matrix elements of $U(x))$ in the character expansion.

3. Solution of the constraints in terms of new dual variables.

Performing the first step one finds for the PF

$$
Z_{\Lambda}(\beta)=\prod_{l \in \Lambda}\left[\sum_{\{r(l)\}} \sum_{m(l), k(l)=1}^{d(r)} C[r(l)]\right] \prod_{x \in \Lambda} Q(x)
$$

where $l=\left(x, e_{n}\right)$ denotes links of the lattice and $Q(x)$ is a group integral. Let $D_{r}^{m k}[U]$ be a matrix element of $U$ in representation $r$. Then $Q(x)$ can be written as

$$
Q(x)=\int D U \prod_{n=1}^{D}\left[D_{r\left(x, e_{n}\right)}^{m\left(x, e_{n}\right) k\left(x, e_{n}\right)}[U] D_{r\left(x-e_{n}, e_{n}\right)}^{m\left(x-e_{n}, e_{n}\right) k\left(x-e_{n}, e_{n}\right)}\left[U^{\dagger}\right]\right]
$$

The second step in the most general settings (for arbitrary graph and for all $G$ ) has been accomplished in [3]. Through the rest of this subsection we consider only the simplest cases of $G=U(1)$ and $G=S U(2)$ in two dimensions. These examples already show a marked difference between abelian and non-abelian models, on the one hand. On the other hand, we want to have relatively simple formulae which can be compared with our formulations. For the $U(1)$ model we have

$$
Q(x)=\delta\left(\sum_{n=1}^{2}\left[r\left(x, e_{n}\right)-r\left(x-e_{n}, e_{n}\right)\right]\right)
$$

where $\delta(x)$ is the Kronecker delta. In $S U(N)$ case the result for $Q(x)$ in 3 is expressed in terms of the Haar intertwiners. We re-state this result in more familiar form expanding $Q(x)$ in the Clebsch-Gordan (CG) series

$$
Q(x)=\sum_{J, s, t} \frac{1}{d(J)} C_{r_{1} m_{1} r_{2} m_{2}}^{J s} C_{r_{1} k_{1} r_{2} k_{2}}^{J t} C_{r_{3} m_{3} r_{4} m_{4}}^{J t} C_{r_{3} k_{3} r_{4} k_{4}}^{J s},
$$

where $r_{1}=r\left(x, e_{1}\right), r_{2}=r\left(x, e_{2}\right), r_{3}=r\left(x-e_{1}, e_{1}\right), r_{4}=r\left(x-e_{2}, e_{2}\right)$ and similar notations are used for $m_{i}$ and $k_{i}$.

The third step can be readily accomplished for the $U(1)$ model. We skip all the details of the derivation which are well known and can be found, for example in [1, 2]. The result for the PF reads

$$
Z_{\Lambda}^{X Y}(\beta)=\sum_{r(x)=-\infty}^{\infty} \prod_{\substack{\star \\ l \in \Lambda}} \Xi_{0}^{X Y}(l)
$$


where the product runs over all links of the dual lattice $\stackrel{\star}{\Lambda}$ and the dual Boltzmann weight is given by

$$
\Xi_{0}^{X Y}(l) \equiv \Xi_{0}^{X Y}\left(r(x)-r\left(x+e_{n}\right) ; \beta\right)=\int_{0}^{2 \pi} \frac{d \phi}{2 \pi} \exp \left[\beta H(\phi)+i\left(r(x)-r\left(x+e_{n}\right)\right)\right]
$$

which in the case of the standard choice $H=\cos \phi$ leads to

$$
\Xi_{0}^{X Y}(l)=I_{r(x)-r\left(x+e_{n}\right)}(\beta)
$$

with $I_{n}(x)$ - the modified Bessel function.

Unfortunately, situation is much more difficult for non-abelian case. $Q(x)$ for $S U(2)$ contains not just one but 4 conditions: 2 Kronecker delta's which constrain magnetic numbers $m_{i}$ and $k_{i}$

$$
m_{1}+m_{2}=k_{3}+k_{4}=s, m_{3}+m_{4}=k_{1}+k_{2}=t
$$

and 2 triangular conditions on representations

$$
\left|r_{1}-r_{2}\right| \leq J \leq r_{1}+r_{2},\left|r_{3}-r_{4}\right| \leq J \leq r_{3}+r_{4}
$$

We are not aware of any attempts in the literature to resolve these constraints simultaneously for all lattice sites. Of course, this does not cause much problems for the strong coupling expansion where only lowest values of $r(l)$ contribute. But situation becomes hopeless in the low temperature region where probably all $r(l)$ become relevant. Eventually, this reduces to the problem of finding the appropriate summation technique when $\beta \rightarrow \infty$ which we could not solve. Moreover, $Q(x)$ as defined in (7) is not strictly positive quantity: on a number of configurations it is negative. We do not know how to perform resummation over the magnetic numbers to get positive Boltzmann weight. Most probably, only complete summation over all magnetic numbers will produce positive quantity. One of the possible ways of how to proceed further is to go over to the triangular lattices identifying representations with the length of the sides of triangles and magnetic numbers with the orientation of the triangles, and we have undertaken some attempts to go along this line. Detailed description of this procedure is however beyond the scope of the present paper.

\subsection{Link formulation of principal chiral models}

We turn now to a different approach to duality transformations based on so-called link representation for partition and correlation functions. That is, the three steps formulated in the beginning of the previous subsection are replaced by the following ones

1. Change of variables in the partition function (3)

$$
V(l)=U(x) U^{\dagger}\left(x+e_{n}\right) .
$$

Integration over original degrees of freedom. This integration generates a set of constraints on the link matrices $V(l)$ known as the Bianchi identities. 
2. Implementation of constraints into the partition function by making use of the invariant delta-function on the group. This step introduces new degrees of freedom which can be associated with plaquettes of the original lattice and which label the irreducible representations of the group $G$. At this step one can go over to the dual lattice so that the new variables belong to the elements of the dual lattice. Integration over link variables. These two steps give already some dual version of the principal chiral model.

3. Before integration over link variables one can decouple summations over matrix indices employing certain orthogonality relations for the CG coefficients (or for some other objects) of the group and multiplying the group matrices entering Bianchi identity in some specially ordered way. Though details depend on the dimension of the lattice the result is that only independent dual variables remain in the theory and their interaction is confined to a subset of all hypercubes of the dual lattice.

The first step of this program has been accomplished twenty years ago in [4]. We therefore restrict ourselves only to the description of the result. The PF (3) can be exactly reformulated in terms of link variables as (with periodic BC)

$$
Z_{\Lambda}(\beta)=\int \prod_{l \in \Lambda} d V(l) \exp \left[\beta \sum_{l \in \Lambda} H[V(l)]\right] J[V],
$$

with Jacobian $J[V]$ given by

$$
J[V]=\prod_{p \in \Lambda} J[V(p)] \prod_{n=1}^{D} J[H(n)],
$$

where $J[V(p)]$ and $J[H(n)]$ are given by

$$
\begin{gathered}
J[V(p)]=\sum_{\{r\}} d(r) \chi_{r}\left(\prod_{l \in p} V(l)\right), \\
J[H(n)]=\sum_{\{r\}} d(r) \chi_{r}\left(\prod_{l_{n}=1}^{L} V\left(l_{n}\right)\right), l=\left(x, e_{n}\right) .
\end{gathered}
$$

$\prod_{p}$ is a product over all plaquettes of lattice $\Lambda$, the sum over $\{r\}$ is sum over all representations of $G=U(N), S U(N), d(r)=\chi_{r}(I)$ is the dimension of $r$-th representation. The group character $\chi_{r}$ depends on a product of the link matrices $V(l)=V_{n}(x)$ along a closed path (plaquette in our case):

$$
\prod_{l \in p} V(l)=V_{n}(x) V_{m}\left(x+e_{n}\right) V_{n}^{\dagger}\left(x+e_{m}\right) V_{m}^{\dagger}(x) .
$$

The expression $\sum_{r} d_{r} \chi_{r}\left(\prod_{l \in p} V(l)\right)$ is the invariant group delta-function which reflects the fact that the product of $U(x) U^{\dagger}\left(x+e_{n}\right)$ around plaquette equals $I$

$$
\prod V(l)=I .
$$


This constraint is called local Bianchi identity while the constraint on holonomy operators

$$
\prod_{l_{n}=1}^{L} V\left(l_{n}\right)=I
$$

is usually called global Bianchi identity. The only solution of two constraints (19) and (20) is a pure gauge (13) which restores the equivalence of the standard and the link formulations. On the lattice with free BC the global constraint should be omitted. Furthermore, in the thermodynamic limit (TL) the contribution from the constraints on the holonomy operators vanishes very fast. In the perturbation theory in $2 D$ it vanishes like inverse powers of $L[5]$, and similar behaviour is expected for $D>2$. Since we are eventually interested in the TL we neglect these global constraints (in fact, the presence of global constraints does not cause any principal problems as will be seen from the procedure described below but their absence makes the technical details somewhat simpler). Thus, for both free and periodic BC we work with the PF

$$
Z_{\Lambda}(\beta)=\int \prod_{l \in \Lambda} d V(l) \exp \left[\beta \sum_{l \in \Lambda} H[V(l)]\right] \prod_{p \in \Lambda} J[V(p)] .
$$

The two-point correlation function in representation $j$ takes the form

$$
\Gamma_{j}(x, y)=\left\langle d^{-1}(j) \chi_{j}\left(\prod_{l \in C_{x y}} W(l)\right)\right\rangle
$$

where $C_{x y}$ is some path connecting points $x$ and $y$ and $W(l)=V(l)$ if along the path the link $l$ points positive direction and $W(l)=V^{\dagger}(l)$, otherwise. For more details on the link formulation we refer the reader to our paper [5], where we have developed a weak coupling expansion for $S U(N)$ spin models using the link representation.

\subsection{Dual of the link formulation: I}

Here we are going to accomplish the step 2 described in the previous subsection. The idea that the true dual variables in the link formulation are the Fourier conjugate to the local Bianchi identity has been proposed also in [4] but the formulae have been explicitely written only for abelian $U(1)$ model in $2 D$. We thus want to reformulate the model (21) on the dual lattice only in terms of discrete variables that are in our case representations $\{r(p)\}$ and magnetic quantum numbers $m_{i}(p)$. We shall use the following conventions for the dual lattice. $\Omega_{x}$ will denote an object dual to site $x$, i.e. $D$-cell of the dual lattice, $\Omega_{l}-(D-1)$-cell of the dual lattice, $\Omega_{p}-(D-2)$-cell of the dual lattice and so on. By definition, object dual to $\mathrm{k}$-th cell is $(D-k)$-cell. As follows from (21) and from the definition of the character which enters the group delta-function in (16) $\chi_{r}(V)=\sum_{n} V_{r}^{n n}$ on the dual lattice the PF may be written as

$$
Z_{\Lambda}(\beta)=Z_{\star}(\beta)=\sum_{S_{n}(0} \prod_{\Omega}\left[d\left[r\left(\Omega_{p}\right)\right] \sum_{m \cdot /(}^{d\left[r\left(\Omega_{p}\right)\right]}\right] \prod_{\Omega_{l}} \Xi_{0}\left(\Omega_{l}\right)
$$


Due to the trace there are 4 variables $m_{i}$ at each $\Omega_{p}$, thus $i=1,2,3,4$. The dual weight $\Xi_{0}\left(\Omega_{l}\right)$ is given by the following one-link integral

$$
\Xi_{0}\left(\Omega_{l}\right)=\int d V e^{\beta H[V]} \prod_{\nu=1}^{D-1}\left[V_{r\left(\Omega_{p}\right)}^{m_{i} m_{i+1}} V_{r\left(\Omega_{p}^{\prime}\right)}^{\dagger n_{i} n_{i+1}}\right]_{\nu},
$$

where $V_{r}^{m n}$ is a matrix element of $r$-th representation. $(D-2)$-cells $\Omega_{p}$ and $\Omega_{p}^{\prime}$ share $(D-1)$-cell $\Omega_{l} \cdot \prod_{\nu}$ runs over $(D-1)$ such pairs.

Similar form for the two-point correlation function in the representation $j$ reads

$$
\begin{array}{r}
\Gamma_{j}(x, y)=\frac{Z_{\Lambda}^{-1}(\beta)}{d(j)} \sum_{s_{1}}^{d(j)} \ldots \sum_{s_{R}}^{d(j)} \sum_{\left\{r\left(\Omega_{p}\right)\right\}} \prod_{\Omega_{p}}\left[d\left[r\left(\Omega_{p}\right)\right] \sum_{m_{i}\left(\Omega_{p}\right)}^{d\left[r\left(\Omega_{p}\right)\right]}\right] \\
\times \prod_{\substack{\Omega_{l} \\
\Omega_{l} \in C_{x y}}} \Xi_{j}^{s_{i} s_{i+1}}\left(\Omega_{l}\right) \prod_{\substack{{ }^{\star} \\
\Omega_{l} \notin C_{x y}}} \Xi_{0}\left(\Omega_{l}\right),
\end{array}
$$

where $s_{R+1}=s_{1}$ and the link integral on $\Omega_{l} \in \stackrel{\star}{C}_{x y}$ is

$$
\Xi_{j}^{s_{i} s_{i+1}}\left(\Omega_{l}\right)=\int d V e^{\beta H[V]} \prod_{\nu=1}^{D-1}\left[V_{r\left(\Omega_{p}\right)}^{m_{i} m_{i+1}} V_{r\left(\Omega_{p}^{\prime}\right)}^{\dagger n_{i} n_{i+1}}\right]_{\nu} V_{j}^{s_{i} s_{i+1}}
$$

Here, $\stackrel{\star}{C}_{x y}$ is a path dual to the path $C_{x y}$ between points $x, y$ on the original lattice.

To vizualize last formulae let us give expression for the PF in two dimensions with $G=S U(2)$. In $2 D$ we have $\Omega_{x}=p, \Omega_{l}=l$ and $\Omega_{p}=x$. Placing as usually dual sites in the centers of original plaquettes we obtain

$$
Z_{\stackrel{\star}{\Lambda}}^{S U(2)}(\beta)=\sum_{r(x)=0, \frac{1}{2}, 1, \ldots}^{\infty} \prod_{\substack{x \in \Lambda^{\star} \\ m_{i}(x)=-r(x)}}\left[(2 r(x)+1) \prod_{l \in \Lambda}^{r(x)} \Xi_{0}(l),\right.
$$

where the dual weight

$$
\Xi_{0}(l) \equiv \Xi_{0}\left(r(x), m_{i}(x), m_{i+1}(x) ; r\left(x+e_{n}\right), t_{i}\left(x+e_{n}\right), t_{i+1}\left(x+e_{n}\right) ; \beta\right)
$$

becomes

$$
\Xi_{0}(l)=\int d V e^{\beta H[V]} V_{r(x)}^{m_{i}(x) m_{i+1}(x)} V_{r\left(x+e_{n}\right)}^{\dagger t_{i}\left(x+e_{n}\right) t_{i+1}\left(x+e_{n}\right)} .
$$

It is very easy to make an integration in (29) expanding the result into the CG series. One then finds the following representation for the dual weight of $S U(2)$ model

$$
\Xi_{0}(l)=\frac{1}{2 r_{2}+1} \sum_{J, k} C[J] C_{r_{1} m_{1} J k}^{r_{2} t_{2}} C_{r_{1} m_{2} J k}^{r_{2} t_{1}},
$$

where we have denoted $r_{1}=r(x), r_{2}=r\left(x+e_{n}\right), m_{1}=m_{i}(x)$ and so on. For the standard choice $H[V]=\chi_{1 / 2}(V)$ the coefficients $C[J]$ can be computed explicitely

$$
C[J]=\frac{2 J+1}{\beta} I_{2 J+1}(2 \beta) .
$$


For the correlation function in Eq.(25) using the CG expansion one gets

$$
\Xi_{j}^{s_{i} s_{i+1}}(l)=\sum_{J \alpha_{1} \alpha_{2}} C_{r_{1} m_{1} j s_{i}}^{J \alpha_{1}} C_{r_{1} m_{2} j s_{i+1}}^{J \alpha_{2}} \Xi_{0}\left(J, \alpha_{1}, \alpha_{2} ; r_{2}, t_{1}, t_{2} ; \beta\right) .
$$

Last expressions can be compared with the dual of the $U(1)$ model given in (8) and with the standard dual formulation of $S U(2)$ model described by formulae (44) and (17). Certainly, the dual weight for $S U(2)$ model $\Xi_{0}(l)$ as given in (29)-(31) is very close to what we have for the dual weight in $U(1)$ model: 1) dual variables, i.e. representations $r(x)$ and magnetic numbers $m_{i}(x)$, live on sites of the dual lattice; 2 ) the full Gibbs measure is factorized into product of dual weights $\Xi_{0}(l)$ over links of the dual lattice. Unlike the standard dual formulation there is no complicated triangular conditions on representations: triangular constraint on $J$ in (30) is only one-link problem which does not lead to any significant complications. Nevertheless, there is also a difference from the abelian case. As follows from the properties of the CG coefficients we have one constraint on the magnetic numbers on every link, namely summation over $k$ in (30) produces the following condition

$$
m_{i}(x)-m_{i+1}(x)=t_{i+1}\left(x+e_{n}\right)-t_{i}\left(x+e_{n}\right) .
$$

This shows that not all dual variables are in fact independent, and this is the main difference from the $U(1)$ model. There is no simple way to make a change of variables such that one gets only independent variables and keeps the locality of interaction. In the next subsection we present a different way of the construction of the dual theory from the link formulation in such a way that the dual weight depends only on independent dual variables.

We finish this subsection with the brief description of some important features of $\Xi_{0}(l)$ :

- As follows from the properties of the coefficients of the expansion $C[J]$ the series in $J$ in (30) gives directly the strong coupling expansion of the model written in closed and compact form. Much less trivial task is to get weak coupling expansion for $\Xi_{0}(l)$ since all $J$ in the series (30) become relevant.

- On all configurations $\left\{r_{i}, m_{i}, n_{i}\right\} \Xi_{0}(l)$ is strictly positive $\Xi_{0}(l)>0$. Though we could not prove it rigorously this claim is supported by the following facts: 1 ) the first term in the strong coupling expansion is strictly positive, thus at sufficiently small $\beta$ where the series converges very fast $\Xi_{0}(l)$ is positive; 2) the leading term of the asymptotic expansion of $\Xi_{0}(l)$ at large $\beta$ is strictly positive on all configurations; 3$)$ numerical computations of $\Xi_{0}(l)$ on a number of configurations and in a wide region of $\beta$ also support this conclusion (we have checked this statement using Mathematica on more than $10^{4}$ configurations). If $\Xi_{0}(l)>0$ on all configurations this gives a chance for a numerical MonteCarlo simulations of the dual model.

- The dominant contribution to $\Xi_{0}(l)$ at large $\beta$ comes from diagonal components of the rotation matrices, non-diagonal contribution is suppressed roughly as $[(m-n) !]^{-1}$. This is. of course a consequence of the fact that when $\beta \rightarrow \infty$ 
the link matrix performs only small fluctuations around unity. In turn, this property gives a possibility to compute low-temperature asymptotic expansion of $\Xi_{0}(l)$. This will be subject of the next section.

\subsection{Dual of the link formulation: II}

The main idea of the following approach is to introduce the independent dual variables from the beginning, namely to find a representation for the group invariant delta-function in terms of quantities which would be free of any conditions. We explain the realization of this idea for $2 D S U(2)$ model and then show how it can be extended to other groups and to higher dimensions.

We start with the representation for $S U(2)$ matrix elements in which the dependence on the magnetic numbers enters only through the Clebsch-Gordan coefficients $C_{b \beta c \gamma}^{a \alpha}{ }^{4}$

$$
V_{r}^{m n}(\omega, \theta, \phi)=\sum_{\lambda k} C_{r m \lambda k}^{r n} U_{r}^{\lambda k}(\omega, \theta, \phi)
$$

where $U_{r}^{\lambda k}(\omega, \theta, \phi)$ is expressed through the spherical harmonics $\mathrm{Y}_{\lambda k}(\theta, \phi)$ and generalized characters $\chi_{\lambda}^{r}(\omega)$ of $S U(2)$

$$
U_{r}^{\lambda k}(\omega, \theta, \phi)=(-i)^{\lambda} \frac{2 \lambda+1}{2 r+1} \sqrt{\frac{4 \pi}{2 \lambda+1}} \mathrm{Y}_{\lambda k}(\theta, \phi) \chi_{\lambda}^{r}(\omega)
$$

Generalized character of rank $\lambda$ in representation $r$ can be defined through the relation

$$
\chi_{\lambda}^{r}(\omega)=i^{\lambda} \sum_{m=-r}^{r} e^{-i m \omega} C_{r m \lambda 0}^{r m} .
$$

We remind that the invariant measure in this parameterization is

$$
\int d V=\frac{1}{4 \pi^{2}} \int_{0}^{2 \pi} \sin ^{2} \frac{\omega}{2} d \omega \int_{0}^{\pi} \sin \theta d \theta \int_{0}^{2 \pi} d \phi
$$

and the fundamental trace becomes

$$
\operatorname{Tr} V_{1 / 2}=2 \cos \frac{\omega}{2}
$$

The basic formula which we need is the following representation for $S U(2)$ characters

$$
\begin{gathered}
\chi_{r}\left(V_{1} V_{2}\right)=\frac{(2 r+1)}{4 \pi} \int_{0}^{2 \pi} d \varphi \int_{0}^{\pi} d \alpha \sin \alpha \Phi\left(r, \varphi, \alpha ; V_{1}\right) \Phi\left(r, \varphi, \alpha ; V_{2}\right), \\
\Phi(r, \varphi, \alpha ; V)=\sum_{\lambda=0}^{2 r}(-i)^{\lambda} \frac{2 \lambda+1}{2 r+1} P_{\lambda}(t) \chi_{\lambda}^{r}(\omega),
\end{gathered}
$$

\footnotetext{
${ }^{4}$ In what follows we use notations and conventions of Ref. [11 for the Clebsch-Gordan coefficients, spherical harmonics, etc.
} 
where $P_{\lambda}(t)$ is the Legendre polynomial and

$$
t=\cos \theta \cos \alpha+\sin \theta \sin \alpha \cos (\phi-\varphi) .
$$

This representation can be easily proved if one uses orthogonality relations for the spherical harmonics and then the addition theorem for the generalized characters.

As the next step, we divide all sites of the dual lattice into sets of even and odd sites. Site $x=\left(x_{1}, x_{2}\right)$ is even if both $x_{1}$ and $x_{2}$ are even or odd, and is odd if one of $x_{i}$ is odd. Thus, all even sites are surrounded by odd sites and vice-versa. Using cyclic properties of the trace we can couple the four matrices entering local Bianchi identities in two different ways, namely

$$
\chi_{r}\left(V_{x}\right)=\chi_{r}\left[V_{12} V_{34}\right],
$$

if the site $x$ is even and

$$
\chi_{r}\left(V_{x}\right)=\chi_{r}\left[V_{23} V_{41}\right],
$$

if the site $x$ is odd. We introduced here obvious notations

$$
V_{12}=V\left(l_{1}\right) V\left(l_{2}\right), V_{34}=V^{\dagger}\left(l_{3}\right) V^{\dagger}\left(l_{4}\right), V_{23}=V\left(l_{2}\right) V^{\dagger}\left(l_{3}\right), V_{41}=V^{\dagger}\left(l_{4}\right) V\left(l_{1}\right) .
$$

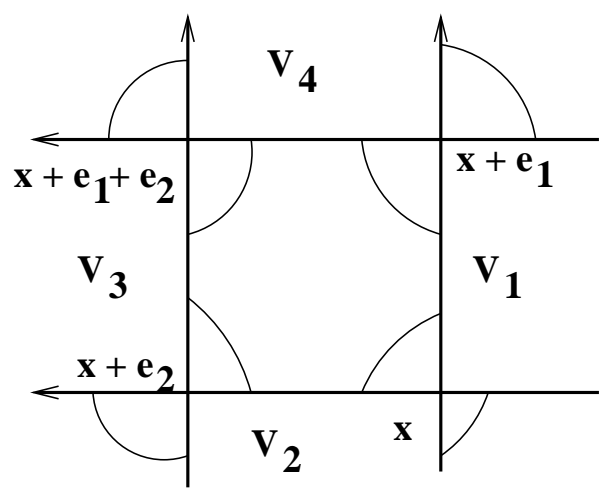

Figure 1: Dual even plaquette and coupling of matrices in group delta's. $V_{i} \equiv V\left(l_{i}\right)$, sites $x$ and $x+e_{1}+e_{2}$ are even, while $x+e_{1}$ and $x+e_{2}$ are odd.

With help of (39) and (42), (43) the Jacobian $J[V]$ defined in (15) and (16) can be written on the dual lattice as

$$
J[V]=\prod_{x, \text { even }} J[V(x)] \prod_{x, \text { odd }} J[V(x)],
$$

where in even sites

$$
J\left(V_{x}\right)=\sum_{r=0,1 / 2, .} \frac{(2 r+1)^{2}}{4 \pi} \int_{0}^{2 \pi} d \varphi \int_{0}^{\pi} d \alpha \sin \alpha \Phi\left(r, \varphi, \alpha ; V_{12}\right) \Phi\left(r, \varphi, \alpha ; V_{34}\right)
$$

while for odd sites

$$
J\left(V_{x}\right)=\sum_{0,0} \frac{(2 r+1)^{2}}{4 \pi} \int_{0}^{2 \pi} d \varphi \int_{0}^{\pi} d \alpha \sin \alpha \Phi\left(r, \varphi, \alpha ; V_{23}\right) \Phi\left(r, \varphi, \alpha ; V_{41}\right)
$$


A set of variables $\xi(x)=\{r(x), \alpha(x), \varphi(x)\}$ is chosen to be dual variables at site $x$. Finally, we decompose all plaquettes of the dual lattice into sets of even and odd plaquettes in such a way that all even plaquettes are surrounded by odd ones and vice-versa. Due to the special coupling of matrices as described above, the integration over link matrices is now coupled only within every, for example even plaquette. This is shown in Fig [1]

Substituting (46) and (47) in (14), re-denoting notations for links $l \in p$ uniformly for all even plaquettes as shown in Fig 1 we obtain dual expression for the partition function

$$
\begin{aligned}
Z_{\stackrel{\star}{\Lambda}}^{S U(2)}(\beta) & =\prod_{x \in \Lambda}\left[\sum_{r(x)=0,1 / 2, \ldots}^{\infty}(2 r(x)+1)^{2} \int_{0}^{2 \pi} \frac{d \varphi(x)}{4 \pi} \int_{0}^{\pi} d \alpha(x) \sin \alpha(x)\right] \\
& \times \prod_{p, \text { even }} B_{0}\left[\xi(x), \xi\left(x+e_{1}\right), \xi\left(x+e_{2}\right), \xi\left(x+e_{1}+e_{2}\right) ; \beta\right],
\end{aligned}
$$

where the dual Boltzmann weight

$$
B_{0}(p) \equiv B_{0}\left[\xi(x), \xi\left(x+e_{1}\right), \xi\left(x+e_{2}\right), \xi\left(x+e_{1}+e_{2}\right) ; \beta\right]
$$

is given by

$$
\begin{aligned}
B_{0}(p) & =\int \prod_{i=1}^{4} d V_{i} \exp \left\{\beta \sum_{i=1}^{4} H\left[V_{i}\right]\right\} \Phi\left(\xi(x) ; V_{1} V_{2}\right) \\
& \times \Phi\left(\xi\left(x+e_{2}\right) ; V_{2}^{\dagger} V_{3}\right) \Phi\left(\xi\left(x+e_{1}+e_{2}\right) ; V_{3}^{\dagger} V_{4}^{\dagger}\right) \Phi\left(\xi\left(x+e_{1}\right) ; V_{4} V_{1}^{\dagger}\right) .
\end{aligned}
$$

Dual expression for the correlation function can be easily re-constructed from Eq.(22) and last formulae. Consider, for simplicity the shortest path between points $x=$ $(0,0)$ and $y=(0, R)$. The corresponding dual path is a set of links which point direction $e_{1}$. Let $|x-y|=R$ be even. Since every link belongs to one and only one even plaquette we obtain

$$
\begin{aligned}
\Gamma_{j}(x, y) & =\frac{Z_{\Lambda}^{-1}(\beta)}{2 j+1} \prod_{x \in \Lambda}\left[\sum_{r(x)=0,1 / 2, \ldots}^{\infty}(2 r(x)+1)^{2} \int_{0}^{2 \pi} \frac{d \varphi(x)}{4 \pi} \int_{0}^{\pi} d \alpha(x) \sin \alpha(x)\right] \\
& \times \prod_{p^{\prime}} B_{0}\left(p^{\prime}\right) \sum_{s_{1}=-j}^{j} \cdots \sum_{s_{R}=-j}^{j} \prod_{p(C)} B_{j}\left(p(C) ; s_{i-1} s_{i} s_{i+1}\right)
\end{aligned}
$$

where $p^{\prime}$ denotes a set of even plaquettes which do not contain links $l \in \stackrel{\star}{C}_{x y}$ and $p(C)$ - a set of even plaquettes which contain such links. In notations of Fig \ we have

$$
B_{j}\left(p(C) ; s_{i-1} s_{i} s_{i+1}\right)=\int \prod_{i=1}^{4} d V_{i} \exp \left\{\beta \sum_{i=1}^{4} H\left[V_{i}\right]\right\} V_{j}^{s_{i-1} s_{i}}\left(l_{1}\right) V_{j}^{s_{i} s_{i+1}}\left(l_{3}\right)
$$


Finally, the disorder operator in the dual representation can be readily obtained from its expression in the link formulation. Let $H[-V(l)]=-H[V(l)]$. Consider the following disorder operator

$$
D(x, y)=\exp \left[2 \beta \sum_{l \in T} H[-V(l)]\right]
$$

where $T=\{l\}=\left[\left(x, e_{1}\right),\left(x+e_{2}, e_{1}\right), \ldots,\left(y-e_{2}, e_{1}\right),\left(y, e_{1}\right)\right]$. Expectation value of this operator can be written as a ratio of two partition functions. With help of the change of variables $V(l) \rightarrow-V(l)$ this ratio can be presented as the following expectation value in the dual formulation

$$
\langle D(x, y)\rangle=\left\langle(-1)^{2 r(x)-2 r(y)}\right\rangle .
$$

Generalization of these representations to higher dimensions is straightforward. In this case we couple link matrices in such a way as to decouple integration over them into a set of integrations within each even $D$-cell of the dual lattice. Then the partition function can be written as

$$
\begin{aligned}
Z_{\star}^{S U(2)}(\beta) & =\prod_{\Omega_{p}}\left[\sum_{r\left(\Omega_{p}\right)=0,1 / 2, \ldots}^{\infty}\left(2 r\left(\Omega_{p}\right)+1\right)^{2} \int_{0}^{2 \pi} \frac{d \varphi\left(\Omega_{p}\right)}{4 \pi} \int_{0}^{\pi} \sin \alpha\left(\Omega_{p}\right) d \alpha\left(\Omega_{p}\right)\right] \\
& \times \prod_{h_{D}, \text { even }} B\left(h_{D}\right),
\end{aligned}
$$

where the dual Boltzmann weight reads

$$
B\left(h_{D}\right)=\int \prod_{\Omega_{l} \in h_{D}}\left[d V\left(\Omega_{l}\right) \exp \left\{\beta H\left[V\left(\Omega_{l}\right)\right]\right\}\right] \prod_{\Omega_{p} \in h_{D}} \Phi\left(\xi\left(\Omega_{p}\right) ; \gamma\left(\Omega_{p}\right)\right) .
$$

Here, $\xi\left(\Omega_{p}\right)=\left\{r\left(\Omega_{p}\right), \alpha\left(\Omega_{p}\right), \varphi\left(\Omega_{p}\right)\right\}$ are dual variables which belong to $(D-2)$-cells of the dual lattice and $\gamma\left(\Omega_{p}\right)=\left\{\omega\left(\Omega_{p}\right), \theta\left(\Omega_{p}\right), \Phi\left(\Omega_{p}\right)\right\}$ are combined angles arising from multiplication of $(D-1)$-cell matrices (original link matrices ) which belong to the same plaquette trace.

To our opinion, the dual representation presented above bears the closest analogy to the abelian case. For example, in two-dimensional model there are three independent dual variables per site as could be expected on general grounds. Further, as follows from the Eq.(50) the interaction between dual variables is local though it is not exactly the same as in abelian models: in addition to nearest-neighbour interaction, there is an interaction over diagonals of even plaquettes. The deficiency of this dual representation is that the dual weight is in general complex valued. This makes the numerical MC computations impossible. On the other hand, we believe it is well suited for an analytical investigation of the model at low temperatures.

In the described above scheme the realization of ideas leading to the local dual formulation relies on the special representation for $S U(2)$ matrices (34), (35). This representation appears to be very efficient at large $\beta$ as we shall show in the next 
section. We do not know if similar parameterization exists for any group $G$. Therefore, for arbitrary group $G$ we proceed as follows. Instead of (39) we use unitarity relations for the CG coefficients of $U(N)$ or $S U(N)$ group [12]

$$
\sum_{\{J k\}} C_{r_{1} m_{1} r_{2} m_{2}}^{J k} C_{r_{1} n_{1} r_{2} n_{2}}^{J k}=\delta_{m_{1}, n_{1}} \delta_{m_{2}, n_{2}}
$$

which allows to present group characters as

$$
\chi_{r}\left(V_{1} V_{2}\right)=\sum_{\{J k\}} G\left(r, J, k ; V_{1}\right) G\left(r, J, k ; V_{2}\right)
$$

where

$$
G(r, J, k ; V)=\sum_{m_{1}, m_{2}} C_{r m_{1} r m_{2}}^{J k} V_{r}^{m_{1} m_{2}}
$$

We then proceed exactly as for $S U(2)$. For the sake of simplicity let us consider the two-dimensional theory. Identifying $\xi(x)=\{r(x), J(x), k(x)\}$ as new dual variables the $\mathrm{PF}$ can be written as

$$
Z_{\substack{\star \\ \Lambda}}^{S U(N)}(\beta)=\sum_{\xi(x)} \prod_{\substack{\star \star \\ x \in \Lambda}} d[r(x)] \prod_{p, \text { even }} B(p),
$$

where the dual Boltzmann weight takes the form

$$
\begin{aligned}
B(p) & =\int \prod_{i=1}^{4} d V_{i} \exp \left\{\beta \sum_{i=1}^{4} H\left[V_{i}\right]\right\} G\left(\xi(x) ; V_{1} V_{2}\right) \\
& \times G\left(\xi\left(x+e_{2}\right) ; V_{2}^{\dagger} V_{3}\right) G\left(\xi\left(x+e_{1}+e_{2}\right) ; V_{3}^{\dagger} V_{4}^{\dagger}\right) G\left(\xi\left(x+e_{1}\right) ; V_{4} V_{1}^{\dagger}\right) .
\end{aligned}
$$

Substituting (59) into the last equation one can express $B(p)$ in terms of one-link integrals defined in (24). Since the $\mathrm{CG}$ coefficients of $S U(N)$ group can be chosen real [12, the dual weight in this representation is also real. We have not studied if it is positive.

\section{$3 \quad 2 D S U(2)$ : Dual weights at low temperatures}

One of the most important application of the dual formulation is the investigation of the low-temperature region of two-dimensional non-abelian models. The first step in this investigation is to establish an asymptotic expansion for dual weights at large $\beta$. Clearly, it is necessary to get asymptotics uniformly valid in all fluctuations of dual variables. It turns out that such asymptotics can indeed be constructed, and this is, in our opinion one of the most important advantages of our dual formulation. Calculation of the asymptotic expansion essentially relies on the fact that when $\beta \rightarrow \infty$ the link matrix performs only small fluctuations around unity both in the finite volume and, most importantly in the thermodynamic limit. Note, that this is not the case for the original $(U(x))$ degrees of freedom: in the large volume limit their fluctuations are not bounded. 


\subsection{Effective theory at low temperatures}

In this subsection we construct an effective low-temperature theory for the dual formulation (48). The corresponding dual weight is given by Eq.(50). We modify it by introducing sources $j_{k}(l)$ which can be related to the correlation function

$$
\begin{aligned}
B_{j}(p) & =\int \prod_{l=1}^{4} d V_{l} \exp \left\{\sum_{l=1}^{4}\left[\beta H\left[V_{l}\right]+j_{k}(l) \omega_{k}(l)\right]\right\} \Phi\left(\xi(x) ; V_{1} V_{2}\right) \\
& \times \Phi\left(\xi\left(x+e_{2}\right) ; V_{2}^{\dagger} V_{3}\right) \Phi\left(\xi\left(x+e_{1}+e_{2}\right) ; V_{3}^{\dagger} V_{4}^{\dagger}\right) \Phi\left(\xi\left(x+e_{1}\right) ; V_{4} V_{1}^{\dagger}\right),
\end{aligned}
$$

where

$$
\omega_{1}(l)=\sin \frac{\omega}{2} \sin \theta \cos \Phi, \omega_{2}(l)=\sin \frac{\omega}{2} \sin \theta \sin \Phi, \omega_{3}(l)=\sin \frac{\omega}{2} \cos \theta .
$$

It is well known (see, e.g. [13]) that when $\beta \rightarrow \infty$ the link matrix performs only small fluctuations around unit matrix. Our next calculations relay on this fact. Let $H[V]$ has a unique maximum at $V=I$. We also require that $H[V]$ possesses character expansion. Then, in the vicinity $V=I$ and in the parameterization (34), (35) one can always construct the following expansion

$$
H\left[V_{l}\right]=H[I]-\frac{\gamma}{2} \sin ^{2} \frac{\omega(l)}{2}+\mathcal{O}\left(\omega^{3}\right) .
$$

For the standard choice $H[V]=\operatorname{Tr} V_{1 / 2}=2 \cos \frac{\omega}{2}$ one finds $H[I]=2$ and $\gamma=2$. Next, we need uniform asymptotic expansion of the function $\Phi(\xi ; V)$. We derive such an expansion in the appendix A. The leading term of this expansion takes the form

$$
\ln \Phi(\xi ; V)=-i \sin \frac{\omega}{2} t \sqrt{R^{2}-1}+\mathcal{O}\left(\sin ^{2} \frac{\omega}{2}\right)
$$

where the classical angular momentum $R$ is introduced in (94). As follows from the semiclassical expansion (101), the remainder is actually bounded as $\mathcal{O}\left(\beta^{-1}\right)$ for all $R$. Therefore, corrections $\mathcal{O}\left(\sin ^{2} \frac{\omega}{2}\right)$ and higher are in fact negligible in the limit $\beta \rightarrow \infty$ so we treat them perturbatively. Lastly, we substitute all these expressions into (62), express combined angles $\omega, \theta, \Phi$ in terms of link angles and perform integration. We omit all these simple but rather cumbersome calculations and give only the final result. Introducing notations

$$
\begin{gathered}
C(\beta)=\exp \left[2 \beta L^{2} H[I]-3 L^{2} \ln (\gamma \beta)\right], \\
R_{k}(x)=\frac{1}{\sqrt{\gamma \beta}} t_{k}(x) \sqrt{R^{2}(x)-1} \\
t_{1}(x)=\sin \alpha(x) \cos \varphi(x), t_{2}(x)=\sin \alpha(x) \sin \varphi(x), t_{1}(x)=\cos \alpha(x)
\end{gathered}
$$

and making the change of variables $R(x)=2 r(x)+1$ so that $R(x)$ takes integer values, we present the result for the dual partition function at large $\beta$ in the form

$$
Z_{\star}(j, \beta)=C(\beta) \prod_{\star}\left[\sum_{R(x)=1}^{\infty} R^{2}(x) \int_{0}^{2 \pi} \frac{d \varphi(x)}{2 \pi} \int_{0}^{\pi} d \alpha(x) \sin \alpha(x)\right] \exp \left[S_{e f f}\right] .
$$


An expression for the effective action $S_{\text {eff }}$ is too large to be given here in full, therefore we present only its expansion. Rescaling sources as $\tilde{j}_{k}(l)=\frac{1}{\sqrt{\gamma \beta}} j_{k}(l)$ the effective action can be expanded as

$$
S_{e f f}=S_{0}+\frac{i}{\sqrt{\gamma \beta}} S_{1}+\frac{1}{\gamma \beta} S_{2}+\mathcal{O}\left(\beta^{-3 / 2}\right)
$$

We have computed first three terms in the above expansion. Here we give $S_{0}$ and $S_{1}$

$$
\begin{gathered}
S_{0}=-\frac{1}{2} \sum_{x, n}\left(R_{k}(x)-R_{k}\left(x+e_{n}\right)+i \tilde{j}_{k}(l)\right)^{2}, \\
S_{1}=\epsilon^{i m k} \sum_{x} W_{i m}(x) R_{k}(x) \\
W_{i m}(x)=p_{i}\left(l_{1}\right) p_{m}\left(l_{2}\right)+p_{i}\left(l_{3}\right) p_{m}\left(l_{4}\right), \text { if } x \text { is even }, \\
W_{i m}(x)=p_{i}\left(l_{1}\right) p_{m}\left(l_{4}\right)+p_{i}\left(l_{3}\right) p_{m}\left(l_{2}\right), \text { if } x \text { is odd } .
\end{gathered}
$$

We have introduced here the following notations

$$
\begin{gathered}
p_{k}\left(l_{1}\right)=R_{k}(x)-R_{k}\left(x+e_{1}\right)+i \tilde{j}_{k}\left(x, e_{1}\right), p_{k}\left(l_{2}\right)=R_{k}(x)-R_{k}\left(x+e_{2}\right)+i \tilde{j}_{k}\left(x, e_{2}\right), \\
p_{k}\left(l_{3}\right)=R_{k}\left(x-e_{1}\right)-R_{k}(x)+i \tilde{j}_{k}\left(x-e_{1}, e_{1}\right), p_{k}\left(l_{2}\right)=R_{k}\left(x-e_{2}\right)-R_{k}(x)+i \tilde{j}_{k}\left(x-e_{2}, e_{2}\right) .
\end{gathered}
$$

Due to the term $R^{2}(x)$ in the measure, summation over $R(x)$ can be extended to include point $R(x)=0$. We use Poisson summation formula, perfrom change of variables $R(x) \rightarrow \sqrt{\gamma \beta} R(x)$ and make the following expansion

$$
R_{k}(x)=\frac{1}{\sqrt{\gamma \beta}} t_{k}(x) \sqrt{\gamma \beta R^{2}(x)-1} \approx t_{k}(x) R(x)+\mathcal{O}\left(\beta^{-1}\right) .
$$

This expansion is valid when $\gamma \beta R^{2}(x)>1$. We call this expansion semiclassical approximation. It allows to define an obvious analog of the vortex-spin-wave representation for the $\mathrm{PF}$

$$
\begin{aligned}
Z_{\star}(j, \beta) & =C(\beta) \prod_{\substack{x \in \Lambda \\
\text { 促 }}}\left[\sum_{m(x)=-\infty}^{\infty} \int_{-\infty}^{\infty} \prod_{k=1}^{3} d R_{k}(x)\right] \\
& \times \exp \left[S_{\text {eff }}\left(R_{k}(x)\right)+2 i \pi \sqrt{\gamma \beta} \sum_{x} m(x)\left(\sum_{k} R_{k}^{2}(x)\right)^{1 / 2}\right] .
\end{aligned}
$$

\subsection{ISO(2)-like approximation}

Here we investigate the dual model given by Eq.(27) with the corresponding dual weight (29). Our approach is to replace the matrix elements in the integrand of (29) by the $I S O(2)$-like matrix elements. Justification of such replacement will be 
given in the course of calculations. For $S U(2)$ matrix elements we use the Wigner $D$-function parametrized by Euler angles

$$
D_{r}^{m n}(\alpha, \omega, \gamma)=e^{-i m \alpha-i n \gamma} d_{r}^{m n}(\omega)
$$

For the sake of simplicity we consider here only the standard action $H[V]=\sum_{n} D_{1 / 2}^{n n}$, i.e. the fundamental character which in this parameterization reads

$$
\sum_{n} D_{1 / 2}^{n n}=2 \cos \frac{\omega}{2} \cos \frac{1}{2}(\alpha+\gamma)
$$

The invariant measure on the group takes the form

$$
\int d V=\frac{1}{16 \pi^{2}} \int_{0}^{4 \pi} d \gamma \int_{0}^{2 \pi} d \alpha \int_{0}^{\pi} d \omega \sin \omega
$$

Substituting last expressions into (29) one can exactly integrate over $\alpha$ and $\gamma$ angles. This gives

$$
\Xi_{0}(l)=\delta_{t_{2}-t_{1}}^{m_{1}-m_{2}} \int_{0}^{\frac{\pi}{2}} d \omega \sin \omega \cos \omega I_{2\left(m_{1}-t_{2}\right)}(2 \beta \cos \omega) d_{r_{1}}^{m_{1} m_{2}}(2 \omega) d_{r_{2}}^{t_{2} t_{1}}(2 \omega) .
$$

To get the asymptotics when $\beta \rightarrow \infty$ we use the fact (described in the previous subsection) that the link matrix in this region performs small fluctuations around unit matrix, namely

$$
\omega \sim \mathcal{O}\left(\beta^{-1 / 2}\right)
$$

It seems to be unfeasible task to solve saddle-point equation for the integrand of (178). We therefore take a different approach to the problem, and replace this integrand by its asymptotics at $\omega \approx 0$ such that

$$
R_{i} \omega \sim \mathcal{O}(1), m_{1}+m_{2}-\text { is arbitrary }, m_{1}-m_{2}-\text { is fixed } .
$$

Here $R_{i}$ is classical angular momentum defined in Eq.94). The last condition states that the main contribution to the integral comes from diagonal components of $S U(2)$ matrix elements, non-diagonal contributions can be treated perturbatively. To be precise, we adjust the following three approximations:

1. We use the following asymptotics for the modified Bessel function

$$
I_{n}(x)=\frac{e^{x}}{\sqrt{2 \pi x}} \exp \left(-\frac{1}{2 x} n^{2}\right)\left(1+\mathcal{O}\left(x^{-1}\right)\right)
$$

when $x \rightarrow \infty$ and such that $n^{2} / x \sim \mathcal{O}(1)$. It leads to

$$
I_{2 s}(2 \beta \cos \omega)=\frac{e^{2 \beta}}{\sqrt{4 \pi \beta}} \exp \left(-\frac{1}{\beta} s^{2}-\beta \sin ^{2} \omega\right)\left(1+\mathcal{O}\left(\sin ^{2} \omega\right)\right) .
$$

Due to the property (79) the remainder is bounded like $\mathcal{O}\left(\beta^{-1}\right)$. 
2. The integration region over $\omega$ is extended to infinity after proper change of variables (this introduces only exponentially small corrections which can be properly bounded).

3. The main approximation concerns the behaviour of matrix elements $d_{r}^{m n}(\omega)$ in the vicinity $\omega \approx 0$. The standard results on the asymptotic expansion of $d_{r}^{m n}(\omega)$ assert that when $\omega \rightarrow 0, r \rightarrow \infty$ the $S U(2)$ matrix elements approach the $I S O(2)$ matrix elements [11, 14]

$$
d_{r}^{m n}(\omega) \asymp J_{m-n}(r \omega),
$$

where $J_{m}(x)$ is the Bessel function. We have found, however that this approximation is oversimplified. In particular, it does not lead to the correct Gaussian distribution of the dual Boltzmann weight that is clearly seen from the effective theory obtained above. Moreover, Eq.(83) states that, e.g. the asymptotics of all diagonal elements is the same. To get more precise asymptotics one can use representation of $d$-function in terms of the Jacobi function. The latter can be treated with help of the Tricomi expansion into degenerate hypergeometric functions as described in [15], section 10.14. Finally, we use uniform Tailor expansion for the degenerate hypergeometric functions [15], section 6.13. This leads to the following asymptotics

$$
d_{r}^{m n}(\omega)=\xi_{m n} J_{k}(R \sin \theta \sqrt{b})+\mathcal{O}\left(\sin ^{2} \omega\right)
$$

where all notations are given in (120). It can be easily checked with help of Mathematica that the last equation gives better approximation to $d$-function compared to Eq.(83), especially at large values of $m+n$ and is valid, in fact for all values of $r$. Moreover, it gives very reasonable approximation in rather wide region of $\omega$, except region $\omega \approx \pi / 2$. In the appendix $\mathrm{B}$ we give independent and simpler proof of this asymptotic expansion calculating also the first correction $\mathcal{O}\left(\sin ^{2} \omega\right)$ to Eq. (84). The final result is given in Eq. (119). We term this asymptotics as $I S O(2)$-like approximation of $S U(2)$ matrix elements. The only (but essential) difference from the exact $I S O(2)$ matrix element is the presence of $\sin \theta$ in the argument of the Bessel function.

Combining Eqs. (82), (84) we arrive finally at the following asymptotic representation for the one-link integral

$$
\Xi_{0}(l)=C(\beta) \delta_{t_{2}-t_{1}}^{m_{1}-m_{2}} \exp \left(-\frac{1}{4 \beta} \alpha^{2}\right) B(l)\left(1+\mathcal{O}\left(\beta^{-1}\right)\right)
$$

where we have used notations

$$
\begin{gathered}
C(\beta)=\frac{e^{2 \beta}}{2 \beta \sqrt{\pi \beta}}, \\
\alpha=m_{1}+m_{2}-t_{1}-t_{2} .
\end{gathered}
$$


Making change of variables $\sin \omega=y / \sqrt{2 \beta}$ in the last integral and extending the integration region over $y$ to infinity the last integral can be written as

$$
B(l)=\int_{0}^{\infty} d y y e^{-\frac{1}{2} y^{2}} J_{m_{1}-m_{2}}\left(\frac{R_{1} \sin \theta_{1}}{\sqrt{2 \beta}} y\right) J_{t_{2}-t_{1}}\left(\frac{R_{2} \sin \theta_{2}}{\sqrt{2 \beta}} y\right),
$$

where $R$ and $\sin \theta$ are defined in Eq.(120). Calculating the last integral we find

$$
\begin{aligned}
\Xi_{0}(l) \asymp C(\beta) \delta_{t_{2}-t_{1}}^{m_{1}-m_{2}} \exp \left[-\frac{1}{4 \beta}\left(R_{1}^{2}\right.\right. & \left.\left.+R_{2}^{2}-2 R_{1} R_{2} \cos \theta_{1} \cos \theta_{2}\right)\right] \\
& \times I_{k}\left(\frac{R_{1} \sin \theta_{1} R_{2} \sin \theta_{2}}{2 \beta}\right),
\end{aligned}
$$

where $k=m_{1}-m_{2}=t_{2}-t_{1}$. Again, we have compared the present asymptotics with the exact expression given by Eq.(30) using Matemathica. Suprisingly, if $\beta$ is sufficiently large the asymptotic formula gives very reasonable approximation for all configurations we have studied. It is interesting to stress that the same asymptotics can be obtained directly from (30), where the CG coefficients are replaced by their semiclassical limit by Ponzano-Regge 16. The method presented above has however two advantages. First of all, we have computed corrections to the leading behaviour (84), therefore $\mathcal{O}\left(\beta^{-1}\right)$-corrections to the asymptotic expansion of one-link integral can be computed in a straightforward manner. Corrections to the Ponzano-Regge formula are in general unknown. Secondly, we find that our method is much simpler.

\section{Discussion}

In this article we proposed dual formulation of non-abelian spin models. Our approach to the dual transformations is summarized in the beginning of the section 2.2. Main formulae of the present paper, Eqs.(23)-(26), (48)-(52) give two versions of the dual formulation. To demonstrate the usefulness of our formulations we have computed the low-temperature asymptotics of the dual Boltzmann weights for two-dimensional model. We reckon that the results for the dual weights provide significant simplification of the partition function at large values of $\beta$. Nevertheless, even in this case the model is still too complicated to be solved exactly. In our next paper [17] we consider some physical applications of the dual representation. In particular, we attempt to give a saddle-point solution of the dual model and derive effective $1 D$ model for the two-point correlation function. Also, we calculate continuum limit of the dual representation and solve classical continuum equations of motion.

Some simple consequences which worth noting can be obtained from (74). Let us perform a shift of variables in the integrand of (74)

$$
R_{k}(x) \rightarrow R_{k}(x)-\frac{i}{2 \gamma \beta} \sum_{l} j_{k}(l) D_{l}(x)
$$

This shift allows to extract the main contribution from the sources to the free energy which emerges from the Gaussian term in the effective action. i.e. $S_{0}$ given bv Ea. ([69) 


$$
Z_{\star}(j, \beta)=C(\beta) \exp \left[\frac{1}{2 \gamma \beta} \sum_{l l^{\prime}} j_{k}(l) G_{l l^{\prime}} j_{k}\left(l^{\prime}\right)\right] \tilde{Z}(j, \beta),
$$

where $\tilde{Z}(j, \beta)$ is a remaining part of the PF. Here $D_{l}(x)$ and $G_{l l^{\prime}}$ are link Green functions introduced in [5]. In [17] we show that the following sources

$$
j_{k}(l)=-2 i \sqrt{j(j+1)} h_{k}, l \in C_{x y}, h_{k}^{2}=1
$$

and $j_{k}(l)=0$, otherwise, can be related to the correlation function in the representation $j$. Substituting these sources into (91) one finds after simple analysis

$$
\Gamma_{j}(R)=Z_{\star}(j, \beta) / Z_{\star}(0, \beta)=\exp \left[-\frac{2}{\gamma \beta} j(j+1) D(R)+\mathcal{O}\left(\beta^{-3 / 2}\right)\right],
$$

where $D(R)=\mathcal{O}(\ln R)$, for $R \rightarrow \infty$. By no means we claim that this result establishes power-like decay of the correlation function: in fact, we do not know the $R$-dependence of the remainder. Therefore, at the moment we can only claim that the shift (90) allows to extract the main perturbative contribution. We would like to stress that the shift (90) is precisely the same as the one used in 22. Unfortunately, we do not know if the methods of [2] can be applied to the partition function (91) to bound the remainder: the essential difference from the $X Y$ model is that even in the absence of vortices both full and effective dual actions are complex. Nevertheless, we think that such possibility deserves further investigations.

\section{A Asymptotics of $\ln \Phi(\xi ; V)$}

In this appendix we calculate the asymptotic expansion of the function $\ln \Phi(\xi ; V)$, where $\Phi(\xi ; V) \equiv \Phi(r, t, \omega)$ is given by Eq.(40)

$$
\Phi(r, t, \omega)=\sum_{\lambda=0}^{2 r}(-i)^{\lambda} \frac{2 \lambda+1}{2 r+1} P_{\lambda}(t) \chi_{\lambda}^{r}(\omega) .
$$

Here, $P_{\lambda}(t)$ is the Legendre polynomial and $t$ is given in Eq.(41). $S U(2)$ matrix is taken in the parameterization (34), (35). Also, we define and compute the semiclassical limit of $\Phi(r, t, \omega)$. To this end we introduce classical angular momentum as

$$
R=2 r+1
$$

We are interested in the expansion of $\Phi(\xi ; V)$ when $\omega \approx 0$ uniformly valid in all variables $\xi$. Because $\varphi$ and $\alpha$ are compact variables the problem of uniformity concerns mainly the value of the variable $r$. We face two situations: 1) $r$ is fixed and 2) $r \rightarrow \infty$. In the second case we look for the asymptotic expansion such that $R \omega \sim \mathcal{O}(1)$.

As the first step we express the generalized characters entering $\Phi(\xi ; V)$ through the associated Legendre functions of the first kind taken on the cut $x \in[-1,1]$

$$
\chi_{\lambda}^{r}(\omega)=\sqrt{\frac{\pi}{2}}\left[\frac{(2 r+1)(2 r+1+\lambda) !}{(2 r-\lambda) ! \sin \underline{\omega}}\right]^{1 / 2} \mathrm{P}_{2 r+1 / 2}^{-\lambda-1 / 2}\left(\cos \frac{\omega}{2}\right) .
$$


When $r$ is fixed, we construct Tailor expansion of $\Phi(\xi ; V)$. It is more convenient to expand in powers of $y=\sin \frac{\omega}{2}$ rather than in powers of $\omega$. From the representation (95) it follows that

$$
\chi_{\lambda}^{r}(\omega)=\frac{\sqrt{\pi}}{2 \Gamma(\lambda+3 / 2)}\left[\frac{R(R+\lambda) !}{(R-\lambda-1) !}\right]^{1 / 2}\left(\frac{y}{2}\right)^{\lambda}\left[1-\frac{R^{2}-(\lambda+1)^{2}}{2 \lambda+3} \frac{y^{2}}{2}+\mathcal{O}\left(y^{4}\right)\right] .
$$

Substituting last expression into Eq.(93), it is straightforward to find the following expansion

$$
\ln \Phi(r, t, \omega)=-i y F_{1}(R, t)+\frac{1}{6} y^{2} F_{2}(R, t)+\frac{i}{6} y^{3} F_{3}(R, t)+\mathcal{O}\left(y^{4}\right)
$$

where the coefficients of the expansion $F_{i}(R, t)$ are

$$
\begin{gathered}
F_{1}(R, t)=t \sqrt{R^{2}-1} \\
F_{2}(R, t)=\left(3 t^{2}-1\right)\left[R^{2}-1-\sqrt{R^{2}-1} \sqrt{R^{2}-4}\right] \\
F_{3}(R, t)=t \sqrt{R^{2}-1}\left\{t^{2}\left[\sqrt{R^{2}-4} \sqrt{R^{2}-9}-\sqrt{R^{2}-1} \sqrt{R^{2}-4}+2\left(R^{2}-1\right)\right]\right. \\
\left.+\frac{3}{5}\left(R^{2}-4\right)-R^{2}+1-\frac{3}{5} \sqrt{R^{2}-4} \sqrt{R^{2}-9}+\sqrt{R^{2}-1} \sqrt{R^{2}-4}\right\}(100)
\end{gathered}
$$

When $R \gg 1$ we expand coefficients $F_{i}$ in powers of $R$. This defines the semiclassical expansion of $\Phi(\xi ; V)$ when $\omega \rightarrow 0, R \rightarrow \infty$ such that $R \omega \sim \mathcal{O}(1)$

$$
\ln \Phi(r, t, \omega)=-i R t y+\frac{i}{2 R} t y+\frac{1}{4} y^{2}\left(3 t^{2}-1-\frac{2 i}{3} R t^{3} y\right)+\mathcal{O}\left(y^{4}\right) .
$$

It would seem that the bound $\mathcal{O}\left(y^{4}\right)$ in (97) and, therefore in (101) does not hold uniformly in $R$. It turns out however that the bound is correct both for fixed $r$ and for $r \rightarrow \infty$. To prove this we need an expansion for the associated Legendre functions at large $r$ uniformly valid in the neighbourhood of the point $\omega=0$. Such an expansion is given by MacDonald's formula [15], section 3.5

$$
\begin{aligned}
\mathrm{P}_{\nu}^{-\mu}(\cos \omega) & =\left[\left(\nu+\frac{1}{2}\right) \cos \frac{\omega}{2}\right]^{-\mu} \\
& \times\left\{J_{\mu}(\alpha)+\sin ^{2} \frac{\omega}{2}\left[\frac{\alpha}{6} J_{\mu+3}(\alpha)-J_{\mu+2}(\alpha)+\frac{1}{2 \alpha} J_{\mu+1}(\alpha)\right]+\mathcal{O}\left(\omega^{4}\right)\right\}
\end{aligned}
$$

where $\alpha=(2 \nu+1) \sin \frac{\omega}{2}$. The ratio of factorials in (95) is expanded as

$$
\left[\frac{(R+\lambda) !}{(R-\lambda-1) !}\right]^{1 / 2}=R^{\lambda+1 / 2}\left[1-\frac{\lambda\left(\lambda^{2}+\frac{3}{2} \lambda+\frac{1}{2}\right)}{6 R^{2}}+\mathcal{O}\left(R^{-4}\right)\right] .
$$

With these results, and using recursion relations for the Bessel functions to reduce order to $\lambda+\frac{1}{2}$ we obtain the representation for the generalized character of the form

$$
\chi_{\lambda}^{r}(\omega)=\left.\sqrt{\frac{\pi}{2}}\left[\frac{R}{2 \sin \underline{\omega}}\right]^{1 / 2} B\left(R, \lambda ; \omega, \partial_{h}\right)\right|_{h=1} \quad J_{\lambda+\frac{1}{2}}(\alpha h)
$$


The function $B(R, \lambda ; \omega)$ has the following asymptotic expansion

$$
B\left(R, \lambda ; \omega, \partial_{h}\right)=1+\frac{\sin ^{2} \frac{\omega}{4}}{3}\left[\frac{7}{4}-\left(\frac{2 \lambda}{\alpha^{2}}(\lambda+1)-\frac{1}{2}\right) \frac{\partial}{\partial h}\right]+\mathcal{O}\left(\sin ^{4} \frac{\omega}{4}\right)
$$

where $\alpha=2 R \sin \frac{\omega}{4}$. Substituting last expressions into Eq. (93) we can easily calculate all the sums and derivatives. This leads to

$$
\begin{aligned}
\ln \Phi(r, t, \omega) & =-2 i R t \sin \frac{\omega}{4}+\frac{i}{R} t \sin \frac{\omega}{4} \\
& +\sin ^{2} \frac{\omega}{4}\left(3 t^{2}-1-\frac{4 i}{3} R t^{3} \sin \frac{\omega}{4}+i R t \sin \frac{\omega}{4}\right)+\mathcal{O}\left(\sin ^{4} \frac{\omega}{4}\right) .
\end{aligned}
$$

Re-expanding last formula in powers of $\sin \frac{\omega}{2}$ one sees that Eq.(106) coincides with Eq. (101). Due to the bound $\mathcal{O}\left(\omega^{4}\right)$ in (102), the same bound holds in (106) and, consequently in (101) and in (97). We thus conclude that the Tailor expansion (97) does provide expansion at small $\omega$ which is valid both for fixed values of $r$ and for $r \rightarrow \infty$.

\section{B Asymptotics of $d_{r}^{m n}(\omega)$}

Here we compute the asymptotic expansion of the $S U(2)$ matrix elements $d_{r}^{m n}(\omega)$ in the classical region $R=(2 r+1) \gg 1$ uniformly valid in the vicinity of the point $\omega=0$ for all allowed values of $m$ and $n, m-n$ is held fixed.

To get such an asymptotics we first present $d$-function in terms of hypergeometric function $F \equiv{ }_{2} F_{1}$

$$
\begin{array}{r}
d_{r}^{m n}(\omega)=\frac{\xi_{m n}}{k !}\left[\frac{(s+k+p) !(s+k) !}{s !(s+p) !}\right]^{\frac{1}{2}}\left(\sin \frac{\omega}{2}\right)^{k}\left(\cos \frac{\omega}{2}\right)^{-p} \\
\times F\left(s+k+1,-s-p ; k+1 ; \sin ^{2} \frac{\omega}{2}\right)
\end{array}
$$

where $\xi_{m n}=1$ if $n \geq m, \xi_{m n}=-1$ otherwise, and

$$
k=|m-n|, p=|m+n|, s=r-\frac{1}{2}(k+p) .
$$

As is seen from the arguments of the hypergeometric function the infinite series in $F$ terminates so that right-hand side of (107) is polynomial in $\sin ^{2} \frac{\omega}{2}$

$$
\begin{aligned}
d_{r}^{m n}(\omega)= & \xi_{m n}\left[A_{k}(x) A_{k}(y)\right]^{\frac{1}{2}}\left(\sin \frac{\omega}{2}\right)^{k}\left(\cos \frac{\omega}{2}\right)^{-p} \\
& \times \sum_{l=0}^{r+\frac{1}{2}(p-k)}(-1)^{l} \frac{\left(\sin ^{2} \frac{\omega}{2}\right)^{l}}{\Gamma(k+1+l) l !} \mathcal{F}_{l}(x, y),
\end{aligned}
$$

where we have introduced the following notations

$$
A_{k}(x)=\frac{\Gamma\left(x-\frac{1}{2} k+\frac{1}{2}\right)}{\Gamma(r+\underline{1} k+\underline{1})}
$$




$$
\begin{aligned}
\mathcal{F}_{l}(x, y) & =\frac{\Gamma\left(x+\frac{1}{2} k+\frac{1}{2}\right)}{\Gamma\left(x-\frac{1}{2} k+\frac{1}{2}-l\right)} \frac{\Gamma\left(y+\frac{1}{2} k+\frac{1}{2}+l\right)}{\Gamma\left(y-\frac{1}{2} k+\frac{1}{2}\right)}, \\
x & =r+\frac{1}{2}(1+p), y=r+\frac{1}{2}(1-p) .
\end{aligned}
$$

The second step consists in expanding the ratio of Gamma functions. This can be done with help of the following formula

$$
\frac{\Gamma(x+a)}{\Gamma(x+b)}=x^{a+b}\left(\sum_{s=0}^{N-1} \frac{(-1)^{s}}{s ! x^{s}} B_{s}^{(a-b+1)}(a)(b-a)_{s}+\mathcal{O}\left(x^{-N}\right)\right)
$$

where $B_{s}^{(y)}(x)$ are the generalized Bernoulli polynomials. Important point concerns the large expansion parameter we use. We take not merely classical angular momentum $r+\frac{1}{2}$ but rather quantities $x$ and $y$ defined above. Such a choice gives more accurate asymptotics valid in a wider region of parameters. Then, in the case of quantity $\mathcal{F}_{l}(x, y)$ the series in (113) terminates because $a-b=k+l$ and representation (113) becomes exact. It leads to

$$
\begin{aligned}
\mathcal{F}_{l}(x, y)=(x y)^{l+k} & \sum_{s_{1}, s_{2}=0}^{l+k} \frac{1}{x^{s_{1}} y^{s_{2}} s_{1} ! s_{2} !} \frac{[(l+k) !]^{2}}{\left(l+k-s_{1}\right) !\left(l+k-s_{2}\right) !} \\
& \times B_{s_{1}}^{(k+l+1)}\left(\frac{1}{2}(k+1)\right) B_{s_{2}}^{(k+l+1)}\left(\frac{1}{2}(k+1)+l\right)
\end{aligned}
$$

what is essentially the desired expansion at large $x$ and $y$. It follows from the last representation that

$$
\begin{array}{r}
\mathcal{F}_{l}(x, y)=(x y)^{l+k}\left[1+\frac{1}{2} l(l+k)\left(\frac{1}{y}-\frac{1}{x}\right)-\frac{l^{2}(l+k)^{2}}{4 x y}\right. \\
\left.+\frac{1}{24}(l+k)(l+k-1)\left(3 l^{2}-l-k-1\right)\left(\frac{1}{x^{2}}+\frac{1}{y^{2}}\right)+\mathcal{O}\left(x^{-3}, y^{-3}\right)\right] .
\end{array}
$$

For $A_{k}(x)$ following the same procedure one finds

$$
A_{k}(x)=x^{-k}\left[1+\frac{1}{24 x^{2}} k\left(k^{2}-1\right)+\mathcal{O}\left(x^{-4}\right)\right] .
$$

Substituting last expressions into (109) we get after some algebra

$$
\begin{array}{r}
d_{r}^{m n}(\omega)=\xi_{m n}\left(\cos \frac{\omega}{2}\right)^{-p} \sum_{l=0}^{\infty} \frac{(-1)^{l}}{(l+k) ! l !}\left(\frac{t}{2}\right)^{k+2 l} \\
\left\{1+\frac{1}{2} l(l+k)\left(\frac{1}{y}-\frac{1}{x}\right)-\frac{l^{2}(l+k)^{2}}{4 x y}+\frac{k\left(k^{2}-1\right)}{48}\left(\frac{1}{x^{2}}-\frac{1}{y^{2}}\right)+\right. \\
\left.\frac{1}{24}(l+k)(l+k-1)\left(3 l^{2}-l-k-1\right)\left(\frac{1}{x^{2}}-\frac{1}{y^{2}}\right)\right\} .
\end{array}
$$


Here we have extended summation over $l$ to infinity since this introduces corrections of the order $\mathcal{O}\left(\omega^{2 r}\right)$ or less. Recalling now the series representation for the Bessel function

$$
J_{k}(t)=\sum_{l=0}^{\infty} \frac{(-1)^{l}}{(l+k) ! l !}\left(\frac{t}{2}\right)^{k+2 l}
$$

we can easily sum up all series in the last formula. Finally, we arrive at the following asymptotic expansion for $d$-function

$$
\begin{array}{r}
d_{r}^{m n}(\omega)=\xi_{m n}\left\{J_{k}(t)+\frac{b}{4}\left[J_{k}(t)+\frac{t}{3 \sin ^{2} \theta}\left(1-2 \cos ^{2} \theta\right)\left(J_{k-1}(t)-\right.\right.\right. \\
\left.\left.\left.J_{k+1}(t)\right)-\frac{1+\cos ^{2} \theta}{6 \sin ^{2} \theta}\left[(k+1) J_{k-2}(t)-(k-1) J_{k+2}(t)\right]\right]+O\left(\sin ^{4} \frac{\omega}{2}\right)\right\} .
\end{array}
$$

We have introduced here the following notations:

$$
R=2 r+1, \cos \theta=\frac{p}{R}, b=\sin ^{2} \frac{\omega}{2}, t=R \sin \theta \sqrt{b} .
$$

\section{References}

[1] R. Savit, Phys.Rev.Lett. 39 (1977) 55; Rev.Mod.Phys. 52 (1980) 453.

[2] J. Fröhlich, T. Spencer, Commun.Math.Phys. 81 (1981) 527.

[3] H. Pfeiffer, J.Math.Phys. 44 (2003) 2891.

[4] G. Batrouni, M.B. Halpern, Phys.Rev. D30 (1984) 1775.

[5] O. Borisenko, V. Kushnir, A. Velytsky, Phys.Rev. D62 (2000) 025013.

[6] O. Borisenko, V. Kushnir, Low-temperature behaviour of $2 D$ lattice $S U(2)$ spin model, Proc. of NATO Workshop "Integrable structures of exactly solvable twodimensional models of quantum field theory", Ed. by S. Pakuliak, and G. von Gehlen, Kluwer Academic Publishers, 2001, 55.

[7] A. Patrascioiu, E. Seiler, J.Statist.Phys. 69 (1992) 573; J.Statist.Phys. 106 (2002) 811-826.

[8] O. Borisenko, P. Skala, Phys.Rev. D62 (2000) 014502.

[9] T. Banks, J. Kogut, R. Myerson, Nucl.Phys. B121 (1977) 493.

[10] M. Göpfert, G. Mack, Commun.Math.Phys. 81 (1981) 97; 82 (1982) 545.

[11] D.A. Varshalovich, A.N. Moskalev, V.K. Khersonskii, Quantum theory of angular momentum, World Scientific Publishing Co.Pte.Ltd., Singapore-New JerseyHong Kong, 1988.

[12] N.Ja. Vilenkin, A.U. Klimyk, Representation of Lie groups and special functions, Kluwer Academic Publishers, Dordrecht-Boston-London, Vol.316, 1995. 
[13] J. Bricmont and J.-R. Fontaine, J.Stat.Phys. 26 (1981) 745.

[14] N.Ja. Vilenkin, Special functions and theory of group representations, Nauka, Moscow, 1991.

[15] H. Bateman, A. Erdelyi, Higher Transcendental Functions, New York-TorontoLondon, 1953; Russian Edition, Nauka, Moscow, Vol.1,2, 1973.

[16] G. Ponzano, T. Regge, Semiclassical limit of Racah coefficients, in Spectroscopic and group theoretical methods in physics, North-Holland Publ. Co., Amsterdam, 1968, 1; K. Schulten, R.G. Gordon, Journal of Math.Phys., V16 (1975), 1971.

[17] O. Borisenko, V. Kushnir, Saddle-point solution of the dual of $2 D S U(2)$ chiral model, in preparation. 